\title{
EXTENSIVE PURPURIC ERUPTIONS IN EPIDEMIC MENINGITIS *
}

\author{
EDWARD A. MORGAN, M.D. \\ NEW YORK
}

Since epidemic meningitis was first described as "spotted fever" a great deal of just criticism has been directed against the term. Even the earliest writers admit that skin manifestations occur in a comparatively small percentage of the cases-too small, indeed, to justify the use of the title.

In his treatise on "A Malignant Epidemic Commonly Called Spotted Fever," North ${ }^{1}$ draws attention to the fact that in the 1806-1807 epidemic in Massachusetts, skin eruptions were very common, while in the later epidemic of 1808-1809 they were almost never observed. "The size of the lesions varied from the head of a pin to a six cent piece and the distribution was more commonly on the face, neck and extremities." He believed that the color of the rash was a guide in prognosis; that is, the darker the shade the more hopeless the outcome.

In the Ireland epidemic in 1867, nearly every case showed skin manifestations. Gordon ${ }^{2}$ reports several such cases and gives a very accurate description of the various forms of eruption.

Frew, ${ }^{3}$ in 1884 , describes seven cases during an epidemic in Scotland, three showing hemorrhagic eruptions; of these three, only one was purpuric. He believed, however, that there were numerous patients showing no eruption who recovered, and these he was not sure were the same disease.

In the Massachusetts epidemic in 1866, Webber ${ }^{4}$ reports that 33 per cent. of the cases showed petechiae; only a few, however, were purpuric. Here also the color of the rash was considered to be an aid in prognosis.

This is in striking contrast to the epidemic in the same state in 1898, during which, according to the investigation by Councilman, Mallory and Wright ${ }^{5}$ only eleven cases out of 111 showed eruption of any kind, and in only one was there a true extravasation into the skin.

* Submitted for publication July 21, 1915.

1. North, Elisha: A Treatise on a Malignant Epidemic Commonly Called Spotted Fever, T. and F. Swords, New York, 1811.

2. Gordon: Dublin Quart. Jour. Med. Sc., 1867, xliii, 408.

3. Frew, William: Glasgow Med. Jour., 1884, xxii, 21.

4. Webber, S. G.: Boston Med. and Surg. Jour., 1866, 1xxv, No. 2, p. 29.

5. Councilman, Mallory and Wright: Report of State Board of Health of Massachusetts, Boston, 1898. 
In a series of fifty cases occurring in New York city reported by Conner $^{6}$ in 1905 , only 5 per cent. showed any eruption, and in a more recent outbreak in Texas, which was so thoroughly investigated by Sophian, ${ }^{7}$ hemorrhagic eruptions were uncommon and true purpura extremely rare, being found in less than 1 per cent. of the cases.

These reports suggest that in the early history of the disease, skin eruptions were more frequent than have been observed recently. This is probably due to the fact that other forms of the disease were not recognized as they are today.

The two following cases are reported as examples of very unusual skin lesions in epidemic meningitis:

\section{REPORT OF CASES}

CASE 1.-F. H., aged two years, was admitted to the Babies' Hospital, Feb. 6, 1914, at 10 a. m.

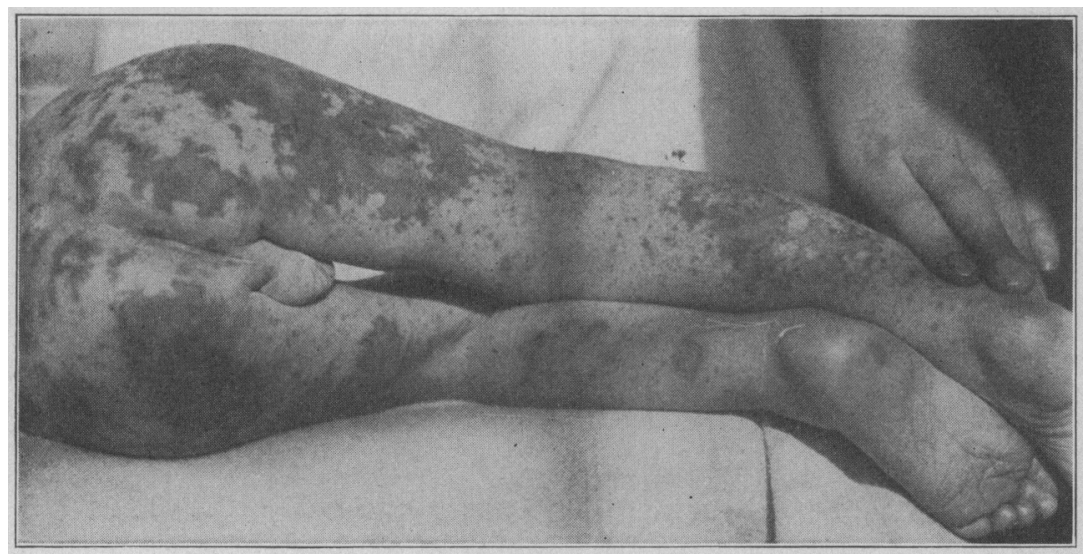

Fig. 1.-Taken on the fourth day of the disease, showing eruption on buttocks and lower extremities, Case 1.

History.--There was no family history of hemophilia or any hemorrhagic disease and the child himself had never been sick before. The patient was well up to twenty-four hours before admission, when he seemed listless, appeared feverish and refused food. The only medication the child had received was twelve tablets of calomel, size unknown. At $11 \mathrm{p}$. m. the same day he seemed better and fell asleep. Two hours later the child awakened screaming and the mother then observed that the face was covered with a black eruption. On removing the bedclothes the eruption was discovered also over the extremities. This, she declared, had not increased up to the time of admission to the hospital. There had been no vomiting, no convulsions, and only slight fever. Stools were green and loose, but not tarry, and the urine was normal in color.

Examination.-On examination was found a well developed and nourished child, evidently severely ill, in a condition of semistupor, with marked pros-

6. Conner, L. H.: Med. News, 1899, 1xxiv, 685.

7. Sophian, Abraham: Epidemic Cerebrospinal Meningitis, C. V. Mosby \& Co., St. Louis, 1913. 
tration. Did not respond to stimuli such as noises; was apparently deaf and blind; periods of drowsiness alternated with periods of restlessness and screaming. The child was extremely hyperesthetic when handled and was particularly so when the legs were moved. No opisthotonos was present but the neck was slightly stiff; knee jerks exaggerated; Kernig's sign, not elicited; pupils equal and reacted normally; no strabismus or conjunctivitis; tache cérébrale present. Respirations rapid but regular; examination of the lungs revealed nothing abnomal. Heart sounds were rapid and slightly feeble; the pulse small and thready, but regular; spleen not enlarged; slight general enlargement of superficial lymphatic glands in cervical, axillary, inguinal and epitrochlear regions; the nodes were soft, small and discrete.

Large blotches of purpura were found on the face, extremities and buttocks interspersed with petechiae of varying sizes. The intervening skin was blanched white and the combination presented an appearance which was ghastly; the child looked as though splashed with a quantity of black ink. The eruption was to some extent symmetrical in that it involved both sides of the

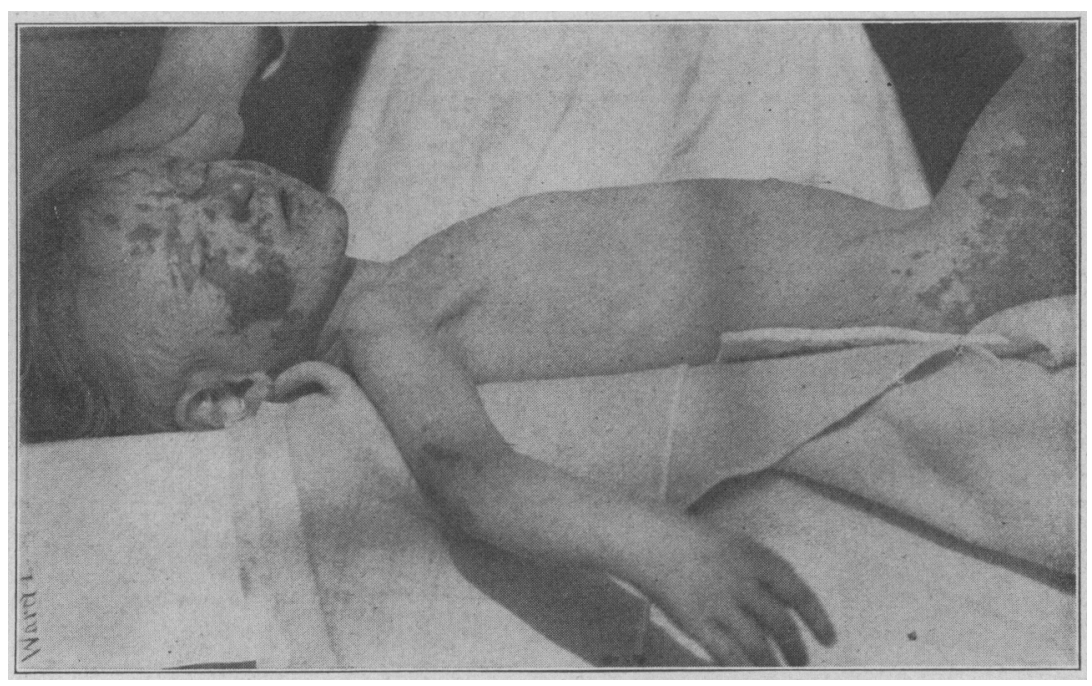

Fig. 2.-Taken on the fourth day of the disease, showing eruption on face and outer side of thighs, Case 1 . Note the absence of lesions on the trunk.

face, both shoulders, the extensor surfaces of the arms, the buttocks and outside of the legs. The largest areas were found on the face, on the elbows, buttocks and thighs, one on the right thigh measuring 6 inches in length. The distribution did not appear to correspond to any particular nerve or vascular supply, but the fact that it was extensive over the elbows and buttocks suggested a possible traumatic etiology. The trunk, except for a few small petechiae, remained clear. The conjunctivae and the mucous membrane of the mouth and throat showed no eruption. The center of the larger patches was purple in color, the periphery was reddish brown, and surrounding this was a zone of hyperemia. There was a distinct elevation above the normal skin level. In some cases this subcutaneous infiltration seemed to correspond with the edge of the purpuric patch but this was not invariable, as the induration could be felt under normal skin in the immediate neighborhood of the larger hemorrhages. The areas of purpura seemed acutely tender and the skin of the whole body was hyperesthetic. The photographs shown in Figures 1 and 2 were taken on February 8 and give an idea of the widespread distribu- 
tion of the eruption; Figure 2 shows very well the absence of the lesions on the trunk.

Subsequent Course.-The eruption did not spread during the day, but the temperature which on admission had been only $100 \mathrm{~F}$., rose abruptly to 102.8 and the child vomited twice very forcibly. The leukocytes were 40,000 per c.mm. with 75 per cent. polymorphonuclears; hemoglobin 80 per cent.; platelets in moderate numbers found in the stained smear; coagulation time of the blood (specimen taken irom vein and Duke's instrument used) was forty-five minutes; bleeding time, two and one-half minutes. Macroscopic, microscopic and chemical tests failed to show blood in either urine or feces. A slight internal strabismus of left eye developed during the day; ophthalmoscopic examination showed no papilledema and no retinal hemorrhages. Ears normal. Cultures were taken from the blood and from the purpuric patches. Lumbar puncture was performed and 20 c.c. of blood-tinged fluid was obtained which was cultured in various mediums.

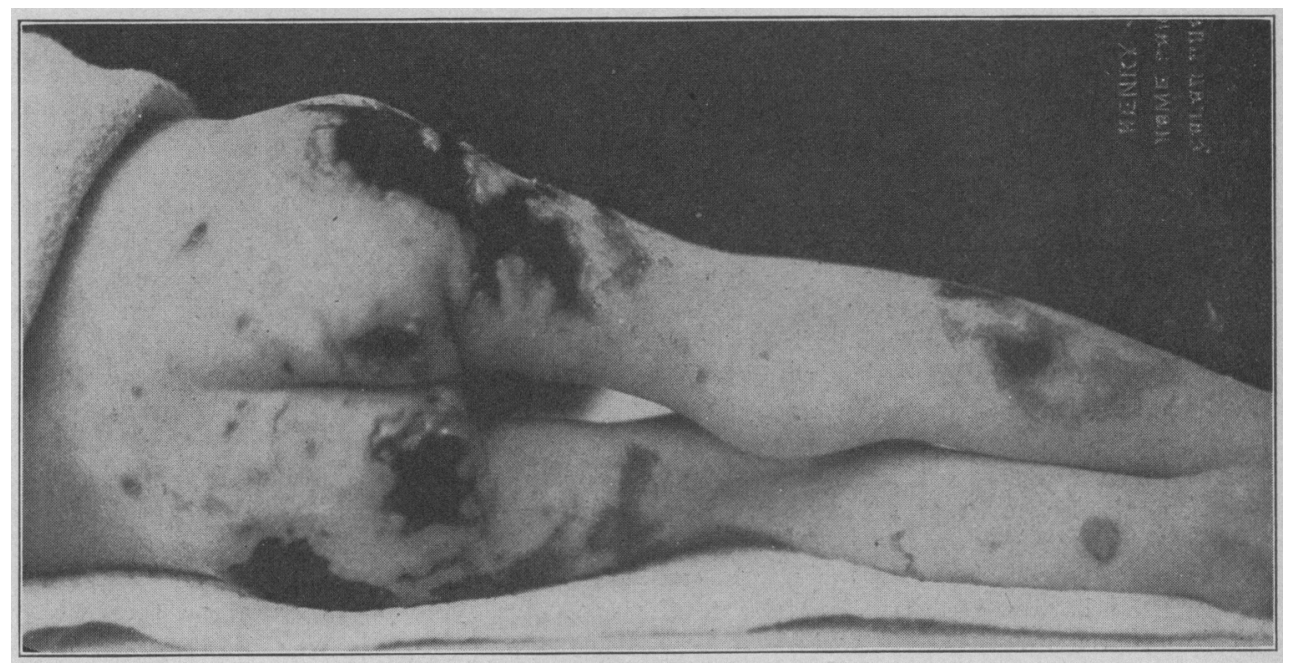

Fig. 3.-Taken on the twelfth day of the disease, Case 1, showing the disappearance of many of the smaller lesions. The commencing separation of the gangrenous tissue is easily discernible at the margin of the larger areas.

The following day the patient was much less irritable and the screaming attacks less frequent. The muscles of the neck and back were more rigid; Kernig's sign absent. Leukocytes 22,000 per c.mm. with 78 per cent. polymorphonuclears; temperature ranging between 101 and 104. Coagulation time of the blood had dropped to twenty minutes; the eruption had not spread or changed in appearance. A second lumbar puncture gave 25 c.c. of turbid fluid, which was removed under increased pressure and showed on examination 4,000 cells per c.mm., of which 92 per cent. were of the polymorphonuclear variety. A direct smear of the fluid showed a very few intracellular and extracellular gramnegative diplococci; 20 c.c. antimeningitis serum were injected intraspinally.

The progress of the case from this point need not be given in detail. The child received intraspinal injections of the serum every day for three days, the size of the injection being controlled by the fluctuations in blood pressure. The cerebrospinal fluid became gradually less turbid; the cell count dropped to 350 per c.mm. on the sixth day of the disease and to 86 per c.mm. on the ninth day. 
The leukocytes were estimated every day and ranged between 16,000 per c.mm. and 20,000 per c.mm. with a gradual drop in the polymorphonuclear percentage. The blood culture taken on February 6 showed in thirty-six hours a growth of meningococci; the organisms could not, however, be demonstrated in the culture taken on the following day. Spinal fluid cultures showed a faint growth of the same organism. Cultures from the purpuric patches remained sterile four days.

The mental condition of the child gradually improved, the vomiting ceased and the rigidity of the neck diminished. A transient right facial paralysis was noticed on February 9, which disappeared on the following day. The temperature after the eighth day of the disease ranged between 100 and $101 \mathrm{~F}$. until the termination of the case.

The course of the eruption was so unusual as to deserve a more detailed description.

The rash on the fifth day of the disease showed a gradual change in color, the center of the larger areas became paler and the outside rim took on a rosecolored tint. On the sixth day one or two of the larger patches showed a central area, grayish-black in color, which had a consistency like cardboard. On the ninth day the smaller purpuric patches began to desquamate, leaving a depressed pigmented scar. The petechiae had also disappeared, leaving only a brown pigmentation. Two days later sloughing commenced at the margin of several of the larger areas of ecchymosis on the body, the excavation in some places extending down to the muscle. The child's breath became very foul due to a separation which had commenced at the left margin of the ecchymosis on the nose. The necrosis penetrated to the nasal passages and through the sinus there was a profuse discharge of fetid mucopus, which was of a very foul and typically gangrenous odor. The photograph shown in Figure 3 was taken February 16 and illustrates the commencing slough at the upper margin of the lesion on the right thigh and hip. Comparison of Figures 1 and 3 shows the disappearance of the petechiae and smaller purpuric spots.

The necrosis continued until the gangrenous tissue had in some localities completely sloughed out leaving a large ulceration lined with unhealthy granulations. The entire tip of the nose became necrotic on the fifteenth day and was removed, exposing the nasal bones. The character of the lesions at this period bore a striking similarity to the extensive symmetrical gangrene seen in severe cases of Raynaud's disease.

The child's condition became progressively worse, the symptom complex being one of general sepsis. The temperature rose to 106.5 on February 22, the seventeenth day of the disease, and the child died the same day. Repeated convulsions occurred during the twenty-four hours previous to death; an exacerbation of the meningitis was stispected, but the cerebrospinal fluid on February 20 was found to be normal.

Necropsy.-An autopsy was performed on February 22 by Dr. Wollstein. With the exception of a superficial strip of bronchopneumonia along the posterior border of the right lower lobe, and a small hemorrhage on one cusp of the aortic valve, the thoracic and abdominal organs were normal. The pia mater at the base of the brain was turbid, but there was no exudate; the vessels of the pia everywhere were congested, most markedly so over the left cortical surface. The ventricles were not distended and contained a small amount of very slightly turbid fluid; the brain substance was congested. There was congestion of the pia mater along the entire length of the spinal cord but no sign of exudate. In short, the findings were those of a recovered case of epidemic meningitis.

CASE 2.-M. O., female, aged 15 months, was admitted to Babies' Hospital, June 11, 1915. 
History.-A previously healthy child except for an attack of pneumonia in January, 1915, and measles in April, 1915; both diseases terminated without complication after running a perfectly normal course.

The mother's story was that on June 8 the child became abruptly ill with vomiting and fever. About eighteen hours later a rash suddenly appeared on the face and extremities; the mother declared she "could watch the spots coming out." The vomiting continued up to admission and the stools were loose and dark green in color. The infant had been listless, but there had been no convulsions.

Examination.-Examination revealed abnormalities only in the nervous and cutaneous systems. The child was slightly apathetic, irritable when handled and markedly hyperesthetic; acutely ill but not prostrated. Pulse and respirations regular. Very slight spasm on attempted flexion of the head on the chest; Kernig's sign absent; patellar reflexes, not exaggerated; tache cérébrale marked; ocular movements normal.

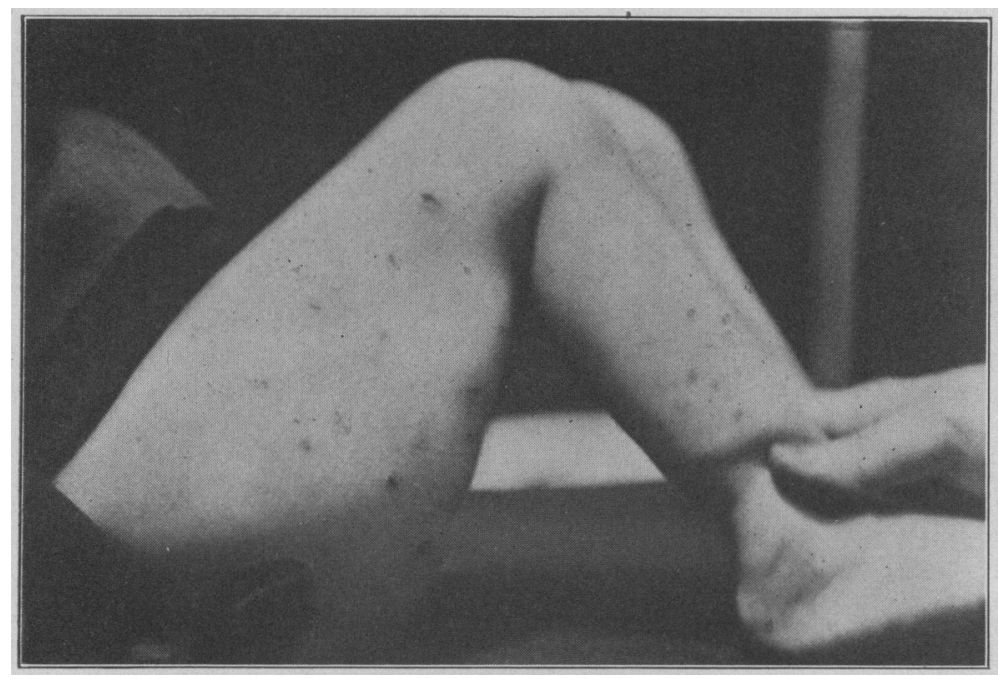

Fig. 4.-Taken on the eighth day of the disease, Case 2, showing eruption over outer surface of legs and thighs.

Scattered over the face, the upper and lower extremities and the buttocks was a hemorrhagic eruption. The majority of the lesions were purpuric, only a few petechiae being present. The spots were of various sizes and shapes, the largest being $13 \mathrm{~mm}$. in length. On the extremities the lesions were found more commonly on the extensor surfaces. The trunk except for a few small spots over the scapular region was clear. The lesions were discrete and the larger ones elevated above the skin level, with a marginal induration of the subcutaneous tissues. The mucous membranes of the mouth and throat were normal; one small hemorrhage found on left conjunctiva and several retinal hemorrhages were revealed by an ophthalmoscopic examination. The larger ecchymoses had a purple center surrounded by. a rose red areola, the intervening skin being much paler than normal.

The character, color and distribution of the eruption bore so striking a resemblance to the eruption in Case 1 as to suggest, in spite of the absence of the usual signs of meningeal involvement, the diagnosis of epidemic meningitis. 
This diagnosis was corroborated by examination of the cerebrospinal fluid. Fifteen cubic centimeters of purulent fluid were obtained under increased pressure; the cell count was 22,500 per c.mm., 88 per cent. of the cells being of the polymorphonuclear variety. A direct smear of the sediment showed moderate numbers of gram-negative diplococci.

Course.-The progress of the case from this point may be briefly summarized: The child received 15 c.c. antimeningitis serum intraspinally on four successive days; the cells in the cerebrospinal fluid showed a gradual diminution in number, so that on June 15 the count was only 660 per c.mm. with 10 per cent. polymorphonuclears, and on June 19, 107 per c.mm. with 2 per cent. polymorphonuclears. Positive cultures of meningococci were obtained from the fluid taken at the first and fourth punctures. Blood culture obtained June 12

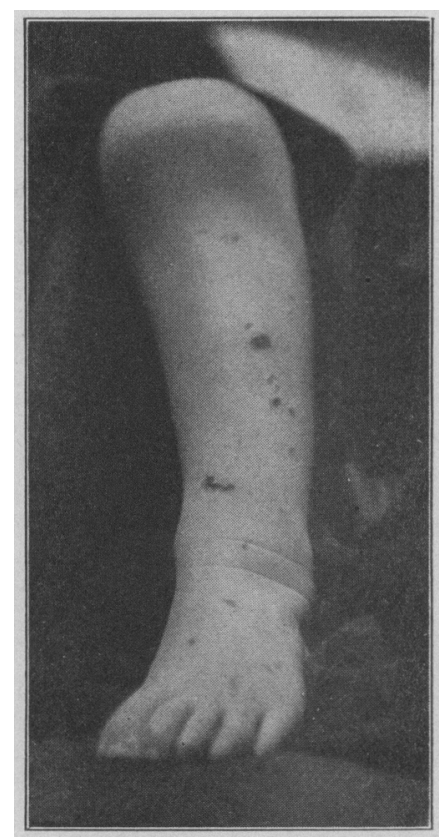

Fig. 5.-Taken on the eighth day of the disease, Case 2, showing a few of the larger lesions on left leg.

also showed a sparse growth of the same organism. The leukocyte count on June 12 was 18,500 per c.mm. with 80 per cent. polymorphonuclears; with each succeeding day there was a drop in the polymorphonuclear percentage, but the total count continued to range between 16,000 and 23,000 per c.mm.

The general improvement of the patient was immediate and striking, rigidity of the neck disappeared and she was able to sit up in bed unassisted six days after admission. The petechiae on June 21 had almost disappeared, nothing being visible but a faint brown pigmentation. Absorption of the subcutaneous hemorrhage around the larger areas was complete by June 21, and the lesion was at this time represented by a depressed scar capped by a reddish brown crust. Figures 4 and 5 are from photographs taken after the smaller lesions had almost disappeared and show a few of the larger individual lesions.

The child's recovery was complete and uncomplicated, she was discharged on June 30 and has continued to improve up to the time of writing. 


\section{DISCUSSION}

Unusual features of the two cases with additional facts of interest:

1. The character and distribution of the eruption, which was unlike the usual purpura hemorrhagica and which though differing greatly in degree was almost identical in both patients.

2. Paucity of other meningeal signs, such as opisthotonos and other manifestations of increased muscle spasm.

3. Demonstration of the specific organism in the blood of both patients, though attempts to grow the same organism from the skin lesions were unsuccessful.

4. Refutation of the old theory that the extent and color of the eruption was of prognostic aid. In both the above cases the ineningeal manifestations were no more marked than usual, and were quite amenable to treatment.

5. Both cases were sporadic as far as could be learned by investigation of the locality and by a study of the Board of Health daily bulletin.

I am indebted to Dr. Holt, on whose service the cases were observed, for permission to publish this report. 